\title{
High energy angular distribution measurements of the exclusive deuteron photodisintegration reaction
}

E. C. Schulte, ${ }^{1,2}$ A. Afanasev, ${ }^{3,4}$ M. Amarian, ${ }^{5}$ K. Aniol, ${ }^{6}$ S. Becher, ${ }^{7}$ K. Benslama, ${ }^{8}$ L. Bimbot,${ }^{9}$ P. Bosted, ${ }^{10}$ E. Brash ${ }^{8}$ J. Calarco ${ }_{11}^{11}$ Z. Chai, ${ }^{12}$ C. Chang, ${ }^{13}$ T. Chang, ${ }^{1}$ J. P. Chen, ${ }^{3}$ S. Choi, ${ }^{14}$ E. Chudakov, ${ }^{12}$ S. Churchwell,,${ }^{15}$ D. Crovelli, ${ }^{16}$

S. Dieterich, ${ }^{16}$ S. Dumalski, ${ }^{8}$ D. Dutta, ${ }^{12}$ M. Epstein, ${ }^{6}$ K. Fissum,${ }^{17}$ B. Fox,${ }^{18}$ S. Frullani, ${ }^{5}$ H. Gao, ${ }^{12}$ J. Gao, ${ }^{19}$ F. Garibaldi, ${ }^{5}$ O. Gayou, ${ }^{20,21}$ R. Gilman, ${ }^{3,16}$ A. Glamazdin, ${ }^{22}$ C. Glashausser, ${ }^{16}$ J. Gomez, ${ }^{3}$ V. Gorbenko, ${ }^{22}$ J.-O. Hansen, ${ }^{3}$ R. J. Holt, ${ }^{2}$ J. Hovdebo, ${ }^{8}$ G. M. Huber, ${ }^{8}$ C. W. de Jager, ${ }^{3}$ X. Jiang, ${ }^{16}$ C. Jones, ${ }^{19}$ M. K. Jones, ${ }^{23}$ J. Kelly, ${ }^{13}$ E. Kinney, ${ }^{18}$ E. Kooijman, ${ }^{24}$ G. Kumbartzki, ${ }^{16}$ M. Kuss, ${ }^{3}$ J. LeRose,${ }^{3}$ M. Liang, ${ }^{3}$ R. Lindgren,${ }^{25}$ N. Liyanage, ${ }^{3}$ S. Malov, ${ }^{16}$ D. Margaziotis, ${ }^{6}$

P. Markowitz, ${ }^{26}$ K. McCormick, ${ }^{27}$ D. Meekins, ${ }^{28}$ Z.-E. Meziani, ${ }^{14}$ R. Michaels, ${ }^{3}$ J. Mitchell, ${ }^{3}$ L. Morand,${ }^{16}$ C. Perdrisat,${ }^{20}$ R. Pomatsalyuk, ${ }^{22}$ V. Punjabi, ${ }^{29}$ A. Radyushkin,${ }^{3,23}$ R. Ransome, ${ }^{16}$ R. Roche, ${ }^{28}$ M. Rvachev,${ }^{30}$ A. Saha, ${ }^{3}$ A. Sarty, ${ }^{28}$

D. Simon, ${ }^{7}$ S. Strauch, ${ }^{16}$ R. Suleiman, ${ }^{12}$ L. Todor, ${ }^{23}$ P. Ulmer, ${ }^{23}$ G. M. Urciuoli, ${ }^{5}$ K. Wijesooriya,,${ }^{1,2}$ B. Wojtsekhowski, ${ }^{3}$ F. Xiong, ${ }^{12}$ and W. $\mathrm{Xu}^{12}$

${ }^{1}$ University of Illinois at Urbana-Champaign, Urbana, Illinois 61801

${ }^{2}$ Argonne National Laboratory, Argonne, Illinois 60439

${ }^{3}$ Thomas Jefferson National Accelerator Facility, Newport News, Virginia 23606

${ }^{4}$ North Carolina Central University, Durham, North Carolina 27707

${ }^{5}$ INFN/Sanita, Roma, Italy

${ }^{6}$ California State University, Los Angeles, California 90032

${ }^{7}$ University of Georgia, Athens, Georgia 30602

${ }^{8}$ University of Regina, Regina, Sasketchewan, Canada 545 OA2

${ }^{9}$ Institut de Physique Nucléaire, Orsay, France

${ }^{10}$ University of Massachusetts, Amherst, Massachusetts 01003

${ }^{11}$ University of New Hampshire, Durham, New Hampshire 03842

${ }^{12}$ Massachusetts Institute of Technology, Cambridge, Massachusetts 02139

${ }^{13}$ University of Maryland, College Park, Maryland 20742

${ }^{14}$ Temple University, Philadelphia, Pensylvania 19122

${ }^{15}$ Duke University, Durham, North Carolina 27706

${ }^{16}$ Rutgers University, New Brunswick, New Jersey 08854

${ }^{17}$ Lund University, Lund, Sweden

${ }^{18}$ University of Colorado, Boulder, Colorado 80309

${ }^{19}$ California Institute of Technology, Pasadena, California 91125

${ }^{20}$ College of William and Mary, Williamsburg, Virginia 23187

${ }^{21}$ Université Blaise Pascal, Aubière, France

${ }^{22}$ Kharkov Institute of Physics and Technology, Kharkov, Ukraine

${ }^{23}$ Old Dominion University, Norfolk, Virginia 23529

${ }^{24}$ Kent State University, Kent, Ohio 44242

${ }^{25}$ University of Virginia, Charlottesville, Virginia 22901

${ }^{26}$ Florida International University, Miami, Florida 33199

${ }^{27}$ DAPNIA, Saclay, France

${ }^{28}$ Florida State University, Tallahassee, Florida 32306

${ }^{29}$ Norfolk State University, Norfolk, Virginia 23504

${ }^{30}$ American University, Washington, District of Columbia 20016

(Received 19 July 2002; published 17 October 2002)

The first complete measurements of the angular distributions of the two-body deuteron photodisintegration differential cross section at photon energies above $1.6 \mathrm{GeV}$ were performed at the Thomas Jefferson National Accelerator Facility. The results show a persistent forward-backward asymmetry up to $E_{\gamma}=2.4 \mathrm{GeV}$, the highest-energy measured in this experiment. The Hard Rescattering and the Quark-Gluon string models are in fair agreement with the results.

DOI: 10.1103/PhysRevC.66.042201

PACS number(s): 13.75.Cs, 24.85. + p, 25.10. $+\mathrm{s}, 25.20 .-\mathrm{x}$

Among the many challenges facing nuclear physicists is the characterization of nuclear reactions in the few $\mathrm{GeV}$ energy regime. Two primary schemes exist to describe nucleonnucleon interactions. The traditional approach involves meson-baryon degrees of freedom. The second employs the quark-gluon degrees of freedom of quantum chromodynamics (QCD) to describe the underlying processes of nuclear reactions. For deuteron photodisintegration at incident photon energies above $1 \mathrm{GeV}$, the existing traditional mesonbaryon descriptions fail to describe the data [1,2]. This breakdown may suggest that QCD degrees of freedom are becoming more appropriate descriptors of the deuteron photodisintegration reaction in the range of a few $\mathrm{GeV}$. The different models which have been developed to improve the 
understanding of this transition region predict very different behaviors for the angular distribution of the differential cross section at constant energy. This work is a presentation of new high-energy measurements of the angular distribution of the deuteron photodisintegration differential cross section. These measurements are designed to probe the transition region between traditional meson-exchange and perturbative QCD.

Deuteron photodisintegration, $d(\gamma, p) n$, is well suited for studying nuclear reactions in the intermediate energy regime. It is an exclusive reaction in which a large amount of momentum is imparted to the constituents [3]. The $d(\gamma, p) n$ reaction also exhibits scaling at forward angles [4-7] consistent with the constituent counting rules $[8,9]$ at sufficiently high incident photon energy. A transition region seems to exist, however, where neither the traditional meson-exchange models nor perturbative QCD [10] describe the data well. This work is a report on new data taken in this region, $1.6 \mathrm{GeV}<E_{\gamma}<2.4 \mathrm{GeV}$ (total center-of-mass energy squared $(s), 9.5 \leq s \leq 12.5 \mathrm{GeV}^{2}$ ), which provide new information on the angular dependence of the $d(\gamma, p) n$ differential cross section.

The highest-energy measurements of the $d(\gamma, p) n$ differential cross section at backward angles which existed previously were performed only to $E_{\gamma}=1.6 \mathrm{GeV}$ [7]. In order to extract a complete angular distribution, these measurements were combined with forward angle data from independent experiments. In addition, recent preliminary angular distributions are becoming available [11] from Hall $B$ at Jefferson Lab.

Recently a new experiment, E99-008, was performed in experimental hall $A$ at Jefferson Lab (JLab). Experiment E99-008 was designed to continue the investigation of the transition region between traditional meson exchange and QCD. By employing the capability of the Hall A High Resolution Spectrometers (HRS) to span a large angular range in the laboratory $\left(15^{\circ}<\theta_{l a b}<112^{\circ}\right.$ in E99-008), the measurements performed during experiment E99-008 cover both forward and backward angles in symmetric steps about $\theta_{\text {c.m. }}$. $=90^{\circ}$. Several models exist to describe the deuteron photodisintegration differential cross section [12]. They predict one of two general features of the angular distribution at constant energy, either symmetry or asymmetry about $\theta_{\text {c.m. }}$. $=90^{\circ}$.

The Asymptotic Meson Exchange model (AMEC) extends the traditional meson exchange approach into the few $\mathrm{GeV}$ energy region [13-15]. In AMEC the deuteron photodisintegration process is divided into a soft (low-energy) portion and a hard (high-energy) portion. The high-energy piece of the process is then parametrized by a form factor [13-15] consistent with the counting rule prescription of Gross and Keister [16] and normalized to data at $E_{\gamma}=1 \mathrm{GeV}$ [13]. The form factor is evaluated in both the instant-form and lightfront relativistic formulations. While both formalisms produce asymmetric angular distributions about $\theta_{\text {c.m. }}=90^{\circ}$, the light-front formalism gives a more reasonable description of the data [13].

Both the Reduced Nuclear Amplitudes (RNA) model [17] and the Quark Exchange model [18], remove the composite nature of the nucleons in the deuteron by dividing out the nucleon form factors from the scattering amplitude. This reduces the deuteron to a composite object of two pointlike nucleons each of which carries one-half of the total deuteron momentum. Using the dipole formula for the form factors [19],

$$
F_{N}\left(\hat{t}_{i}\right)=\frac{C}{\left(1-\frac{\hat{t}_{i}}{0.71 \mathrm{GeV}^{2}}\right)^{2}},
$$

where $C$ is a normalizing constant (chosen to match the data at $\theta_{\text {c.m. }}=90^{\circ}$ ) and $\hat{t}_{i}$ is the momentum transferred to nucleon $i$, the differential cross section from RNA can be calculated by

$$
\frac{d \sigma}{d \Omega_{\mathrm{c} . \mathrm{m} .}} \sim \frac{1}{\left[s\left(s-m_{d}^{2}\right)\right]^{1 / 2}} \frac{F_{p}^{2}\left(\hat{t}_{p}\right) F_{n}^{2}\left(\hat{t}_{n}\right)}{P_{T}^{2}} f^{2}\left(\theta_{\text {c.m. }}\right) .
$$

In Eq. (2) $f\left(\theta_{\text {c.m. }}\right.$.) is a function which describes the angular distribution of the differential cross section and $P_{T}$ is the transverse momentum of the outgoing proton [17]. Brodsky and Hiller propose a method for describing $f^{2}\left(\theta_{\text {c.m. }}\right.$. by modeling deuteron photodisintegration as the photodisintegration of a polarized meson. This reduces the problem to a quarkantiquark interaction. Keeping only the lowest order QCD diagrams yields a function which is dependent on $\cos ^{2}\left(\theta_{\text {c.m. }}\right)$ [17]. As including this model for $f^{2}\left(\theta_{\text {c.m. }}\right)$ does not improve the RNA description of the data, $f^{2}\left(\theta_{\text {c.m. }}\right)$ is left a constant. This method (RNA), because of the nature of the form factors, predicts a symmetric angular distribution for the differential cross section, at a given photon energy.

The Quark-Gluon String (QGS) model [20,21] is a nonperturbative technique which can be applied to calculate the deuteron photodisintegration differential cross section. In this model the deuteron photodisintegration scattering amplitude is found using a planar diagram describing "the exchange of three valance quarks in the $t$ channel with any number of gluon exchanges between them" [20]. This produces a scattering amplitude which is identified with the nuclear Regge pole and a residue which is normalized to previous data at $\theta_{\text {c.m. }}=36^{\circ}$. By using nonlinear Regge trajectories of logarithmic form the QGS model reasonably describes the energy dependence of the deuteron photodisintegration differential cross section. In order to describe the angular dependence, two cases were studied. In the first case, the assumption is made that only isovector photons are dominant in the deuteron photodisintegration process. This produces a symmetric angular distribution for the differential cross section. The second case includes an interference between isovector and isoscalar photons in the deuteron photodisintegration amplitude. Including the interference term results in an asymmetric angular distribution [20,21].

The Hard Rescattering model (HRM) [22], uses a deuteron wave function to describe the long-range behavior of the deuteron. In HRM the photon couples directly to a quark in a nucleon. This results in the interchange of two quarks between the nucleons and the exchange of a hard gluon. High-energy, large-angle neutron-proton scattering data are 


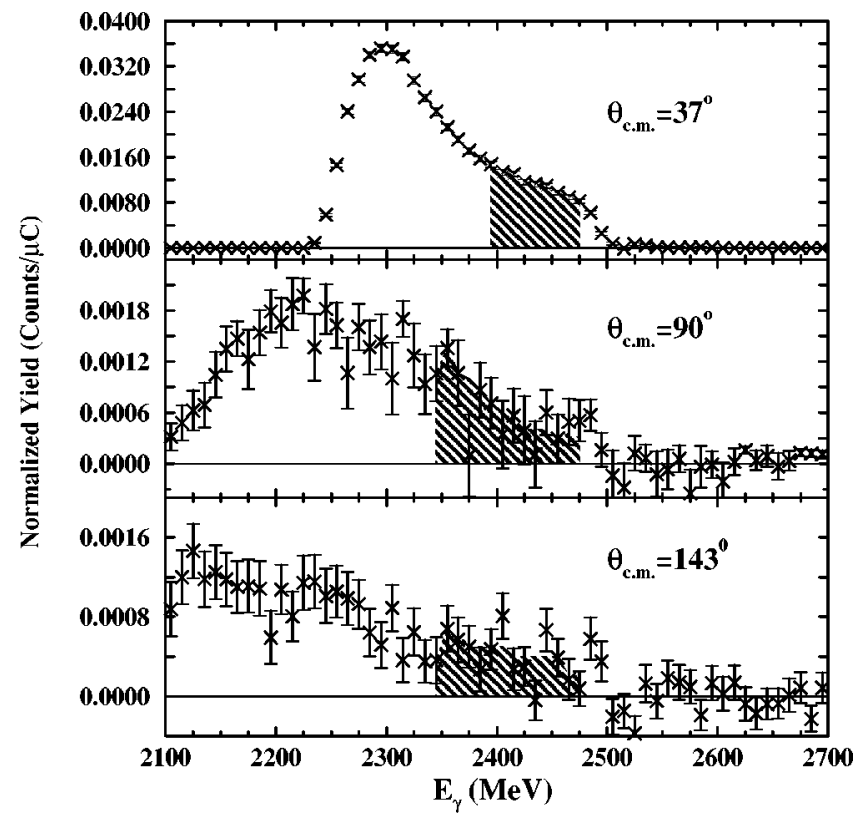

FIG. 1. Reconstructed photon energy spectra after the background-subtraction procedure described in the text has been applied. These spectra are from data taken at a beam energy of $2.5 \mathrm{GeV}$ at center-of-mass angles of $\theta_{\text {c.m. }}=37^{\circ}$ (top panel), $\theta_{\text {c.m. }}=90^{\circ}$ (center panel), and $\theta_{\text {c.m. }}=143^{\circ}$ (bottom panel). The patterned area denotes the region from which the differential cross section was measured.

extrapolated to the appropriate momentum transfer and used to parametrize the quark interchange and gluon exchange. HRM uses a momentum dependent normalization factor determined by previous deuteron photodisintegration data taken at $\theta_{\text {c.m. }}=90^{\circ}$. This normalization is kept for all HRM predictions $[22,23]$.

Jefferson Lab experiment E99-008 was performed in Hall $A$ at the Continuous Electron Beam Accelerator Facility. This experiment used the beam-left High Resolution Spectrometer
(HRSL) to detect recoil protons from the deuteron photodisintegration reaction. The photons were generated by passing high-energy electrons $\left(E_{\text {beam }}=1.670,1.950\right.$, and 2.499 $\mathrm{GeV}$ ) through a $6 \%$ radiation-length copper target. The resultant bremsstrahlung photons, and residual electrons, then irradiated a liquid hydrogen or deuterium target $15 \mathrm{~cm}$ long. The HRSL was chosen for this experiment because of its reasonably large solid angle $\left(\Delta \Omega \approx 5 \mathrm{msr}, \delta \theta_{\text {c.m. }}\right.$. $= \pm 28 \mathrm{mr})$, reasonable momentum acceptance $(\delta=$ $\pm 4.5 \%)$, and ability to achieve the large laboratory angles $\left(\theta_{\text {lab }} \approx 112^{\circ}\right)$ needed in this experiment. The recoil protons were tracked using the HRSL pair of vertical drift chambers. The trigger was formed by a coincidence of the two planes of scintillator hodoscope, provided in the detector package.

A complete angular distribution, with data at angles symmetric about $\theta_{\text {c.m. }}=90^{\circ}$, was recorded at each of three incident photon energies. Data were recorded in one of four configurations: (i) deuterium target, radiator in; (ii) deuterium target, radiator out; (iii) hydrogen target, radiator in; and (iv) hydrogen target, radiator out. Data taken with the radiator out were used to subtract events caused by electrodisintegration of the deuteron by residual electrons which impinged upon the target. The hydrogen data were used to help understand and subtract background.

By conservation of energy and momentum, incident photon energy $E_{\gamma}$ spectra were reconstructed (for example Fig. 1). From these spectra the protons necessary to extract the differential cross section were selected. In order to assure the protons used in the measurement were from the two-body reaction $[\gamma(d, p) n]$, only those protons which were produced near the bremsstrahlung endpoint were used in the analysis. A target length cut was placed on the data for those settings at forward angles where the aluminum target end-caps could be viewed. This provided for the elimination of background events from the target end-caps.

Even after elimination of the aluminum target end-caps, some background events remained, especially at large centerof-mass angles. Background was observed as events which

TABLE I. Center-of-mass cross sections for Jefferson Lab experiment E99-008. The errors shown are first statistical followed by systematic error. Measurements were not made for $\theta_{\text {c.m. }}=26^{\circ}$ at 1.9 and $2.4 \mathrm{GeV}$, due to spectrometer limitations. At $\theta_{\text {c.m. }}=127^{\circ}$ at $2.4 \mathrm{GeV}$, a support post for the target chamber obscured the target making the data unusable.

\begin{tabular}{lccccr}
\hline \hline \multicolumn{5}{c}{ Angular distribution cross sections } & \\
$\theta_{\text {c.m. }}$ & $1.6 \mathrm{GeV}$ & & $1.9 \mathrm{GeV}$ & $2.4 \mathrm{GeV}$ \\
& $\frac{d \sigma}{d \Omega_{\text {c.m. }}}\left(\frac{n b}{s r}\right)$ & $\theta_{\text {c.m. }}$ & $\frac{d \sigma}{d \Omega_{\text {c.m. }}}\left(\frac{n b}{s r}\right)$ & $\theta_{\text {c.m. }}$ & $\frac{d \sigma}{d \Omega_{\text {c.m. }}}\left(\frac{n b}{s r}\right)$ \\
\hline $26.6^{\circ}$ & $19.2 \pm 0.2 \pm 1.2$ & & & \\
$30.3^{\circ}$ & $14.6 \pm 0.3 \pm 0.9$ & $30.3^{\circ}$ & $6.36 \pm 0.08 \pm 0.38$ & $30.4^{\circ}$ & $1.73 \pm 0.03 \pm 0.10$ \\
$37.4^{\circ}$ & $8.72 \pm 0.18 \pm 0.52$ & $37.4^{\circ}$ & $4.02 \pm 0.04 \pm 0.24$ & $37.3^{\circ}$ & $0.89 \pm 0.02 \pm 0.05$ \\
$53.6^{\circ}$ & $4.33 \pm 0.07 \pm 0.26$ & $53.5^{\circ}$ & $1.93 \pm 0.04 \pm 0.12$ & $53.4^{\circ}$ & $0.47 \pm 0.01 \pm 0.03$ \\
$70.7^{\circ}$ & $3.35 \pm 0.07 \pm 0.20$ & $70.6^{\circ}$ & $1.72 \pm 0.01 \pm 0.10$ & $70.5^{\circ}$ & $0.32 \pm 0.01 \pm 0.02$ \\
$90.8^{\circ}$ & $2.58 \pm 0.13 \pm 0.18$ & $90.7^{\circ}$ & $1.08 \pm 0.02 \pm 0.08$ & $90.6^{\circ}$ & $0.18 \pm 0.02 \pm 0.01$ \\
$110.7^{\circ}$ & $2.47 \pm 0.13 \pm 0.18$ & $110.6^{\circ}$ & $1.01 \pm 0.03 \pm 0.07$ & $110.5^{\circ}$ & $0.23 \pm 0.03 \pm 0.02$ \\
$127.6^{\circ}$ & $3.02 \pm 0.12 \pm 0.21$ & $127.0^{\circ}$ & $1.10 \pm 0.09 \pm 0.08$ & & \\
$143.5^{\circ}$ & $4.42 \pm 0.24 \pm 0.51$ & $142.3^{\circ}$ & $1.20 \pm 0.16 \pm 0.14$ & $143.4^{\circ}$ & $0.44 \pm 0.07 \pm 0.05$ \\
\hline \hline
\end{tabular}




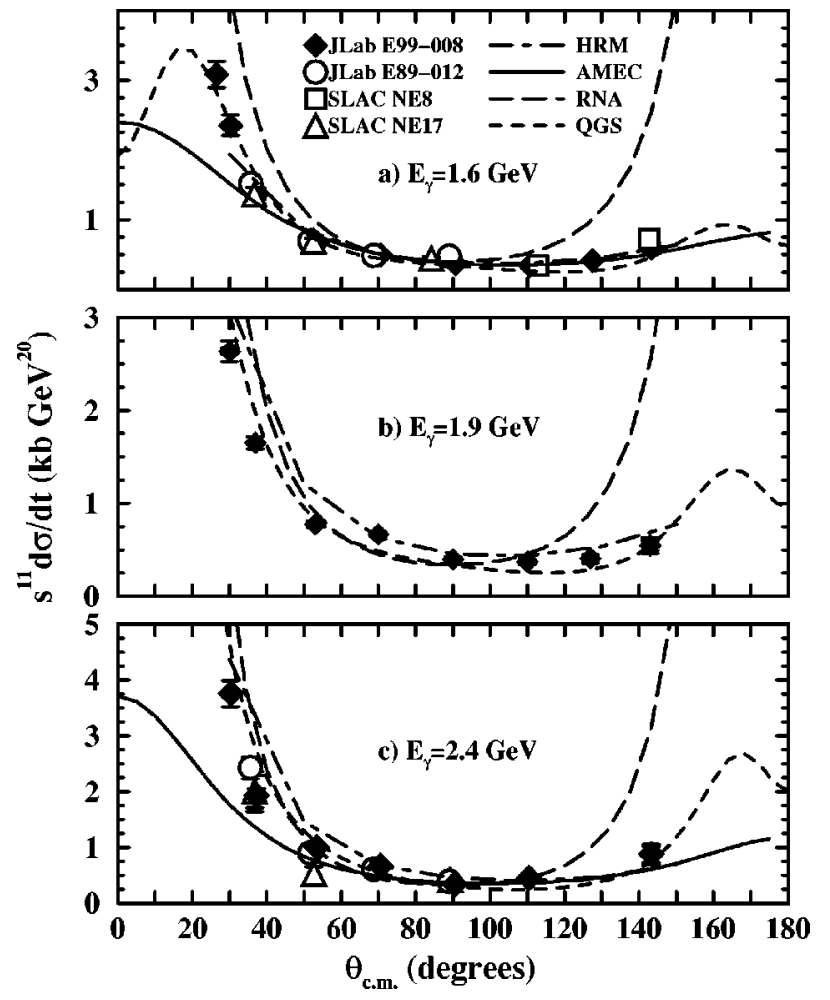

FIG. 2. $s^{11} d \sigma / d t$ for $d(\gamma, p) n$ at (a) $E_{\gamma}=1.6 \mathrm{GeV}$, (b) $E_{\gamma}=1.9 \mathrm{GeV}$, and (c) $E_{\gamma}=2.4 \mathrm{GeV}$. Error limits shown are both statistical and total on JLab and SLAC NE17 data. Errors on SLAC NE8 data are statistical only. Theory curves are discussed in the text (the prediction [23] from HRM is preliminary). The present data are the solid diamonds. Previous data are (circles) JLab E89-012 [6], (triangles) SLAC NE17 [4], and (squares) SLAC NE8 [7].

were reconstructed at kinematically forbidden energies in the photon energy spectra (energies where the reconstructed $\mathrm{E}_{\gamma}$ is greater than the end-point energy). These events seemed to have two causes, photoprotons from internal aluminum target components, and protons produced in multistep processes. Multistep processes account for up to $20 \%$ of the background at large angles. Further background suppression is achieved by scaling the hydrogen data to match the deuterium data in kinematically forbidden regions and subtracting the scaled hydrogen spectra from the deuterium spectra. This is done for both the radiator in and radiator out data. The radiator out data, modified by an energy-dependent factor which compensates for the presence of the bremsstrahlung radiator, is used to eliminate events caused by electroproduction. Background accounted for $30 \%$ of the events collected in data taken at angles $\leqslant 90^{\circ}$ in the center of mass. In the worst case (data taken at $\theta_{\text {c.m. }} \approx 143^{\circ}$ ), background events accounted for more than $80 \%$ of the events taken. High background rates at large backward angles can be attributed to processes such as multistep interactions involving two target deuterons or proton production from aluminum components of the target cell. Also, the cross sections in the laboratory are quite small at backward angles, which makes the measurements more challenging.

Proper normalization of the background-subtracted photo- proton yield was necessary for extraction of the differential cross section. The normalization parameters consisted of the total number of real bremsstrahlung photons which impinged upon the target, the solid angle subtended by the HRSL, the target length, and other corrections, such as proton absorption through the target-spectrometer system and tracking efficiency. The real photon number was calculated using the method of Matthews and Owens for thick targets, and was accurate to $3 \%$ [24]. The HRS solid angle was studied by Monte Carlo simulation. The Monte Carlo was tested against $p\left(e, e^{\prime}\right) p$ scattering data and the agreement was better than $3 \%$. Other corrections applied to the data included $\leqslant 9 \%$ for proton absorption in the target cryogen and spectrometerdetector package, $\approx 1 \%$ was applied for tracking efficiency, and an estimate of $\approx 1 \%$ for electronics dead time.

The total systematic error was estimated to be $\lesssim 12 \%$. Uncertainties in the beam current, beam energy, target length, and photon energy reconstruction were $\lesssim 1.5 \%$ in the center-of-mass differential cross section. Error assigned to the background subtraction gave the dominant contribution, $\approx 10 \%$ in the worst case, to the total systematic error. All other errors including solid angle determination and real bremsstrahlung calculations contributed the remaining $\$ 5.5 \%$. The final center-of-mass differential cross sections are presented in Table I.

Shown in Fig. 2 are the present data at $E_{\gamma}=1.6 \mathrm{GeV}$, $1.9 \mathrm{GeV}$, and $2.4 \mathrm{GeV}$ plotted as $s^{11} d \sigma / d t$ to remove the energy dependence for comparison to previous data. The present data are in good agreement with previous data. While the data show both forward and backward peaking, a persistent forward-backward asymmetry is present in the data up to the highest-energy measured in this experiment $\left(E_{\gamma}=2.4 \mathrm{GeV}\right)$. This asymmetry is not well described by the prediction of the AMEC model $[13,14]$, the solid curve in Fig. 2, in which the photon couples directly to one of the nucleons. The RNA (long-dashed curve in Fig. 2) and quark exchange model $[17,18]$ in which the photon couples to an exchange particle, however, predict a symmetric angular distribution about $\theta_{\text {c.m. }}=90^{\circ}$, clearly not described by the data.

The two remaining models, QGS [20,21] (short-dashed line in Fig. 2) and HRM [22,23] (short-long dashed curve in Fig. 2), also provide predictions about the angular distribution of the $d(\gamma, p) n$ differential cross section. QGS, the Regge phenomenological model, reproduces the differential cross sections fairly well. Both the magnitude and forwardbackward asymmetry are reasonably well described. Preliminary calculations from HRM are presented in Fig. 2 [23]. Like QGS, HRM also describes the data reasonably well, both in magnitude and forward-backward asymmetry.

The data presented in this work are the first complete high-energy angular distribution measurements of the deuteron photodisintegration differential cross section. During Jefferson Lab experiment E99-008, data were recorded at center-of-mass angles symmetrically spaced about $\theta_{\text {c.m. }}=90^{\circ}$, with additional forward angle data taken at $\theta_{\text {c.m. }}=30^{\circ}$. The data present a persistent forward-backward asymmetry, described fairly well by the QGS and HRM models. The asymmetry is still apparent up to the highest energy measured during this experiment, $E_{\gamma}=2.4 \mathrm{GeV}$. 
The authors extend their thanks T.-S. H. Lee for many useful discussions. Thanks are also extended to the staff of the Accelerator Division at Jefferson Lab for the delivery of the high quality electron beam and to the hall A technical staff and Target Groups for their maintenance, installation, and repair of the HRSL and its detectors and the cryotargets. This work was supported by the United States Department of Energy, the National Science
Foundation, the Natural Sciences and Engineering Research Council of Canada, the French Commissariat à l'Energie Atomique and Centre National de la Recherche Scientifique, the Italian National Institute for Nuclear Physics, and the Swedish Natural Science Research Council. This work was supported by DOE Contract No. DE-AC05-84ER40150 under which the Southeastern Universities Research Association (SURA) operates the Thomas Jefferson National Accelerator Facility.
[1] T. S.-H. Lee, Argonne National Laboratory Report No. PHY5253-TH-88.

[2] T. S.-H. Lee, Proceedings of the International Conference on Medium and High Energy Nuclear Physics, Taipei, Taiwan, 1988 (World Scientific, Singapore, 1988).

[3] R.J. Holt, Phys. Rev. C 41, 2400 (1990).

[4] J.E. Belz et al., Phys. Rev. Lett. 74, 646 (1995).

[5] E.C. Schulte et al., Phys. Rev. Lett. 87, 102302 (2001).

[6] C. Bochna et al., Phys. Rev. Lett. 81, 4576 (1998).

[7] S. Freedman et al., Phys. Rev. C 48, 1864 (1993).

[8] S.J. Brodsky and G.R. Farrar, Phys. Rev. Lett. 31, 1153 (1973).

[9] V. Matveev et al., Lett. Nuovo Cimento Soc. Ital. Fis. 7, 719 (1973).

[10] K. Wijesooriya et al., Phys. Rev. Lett. 86, 2975 (2001).

[11] M. Mirazita, hep-ph/0205213.
[12] R. Gilman and F. Gross, J. Phys. G 28, R37 (2002).

[13] A. Dieperink and S. Nagorny, Phys. Lett. B 456, 9 (1999).

[14] S. Nagorny et al., Yad. Fiz. 55, 345 (1992).

[15] A. Dieperink (private communication).

[16] F. Gross and B.D. Keister, Phys. Rev. C 28, 823 (1983).

[17] S.J. Brodsky and J.R. Hiller, Phys. Rev. C 28, 475 (1983).

[18] A. Radyushkin (private communication).

[19] S.J. Brodsky and B.T. Chertok, Phys. Rev. Lett. 37, 269 (1976).

[20] V.Y. Grishina et al., Eur. Phys. J. A 10, 355 (2001).

[21] E. De Sanctis (private communication).

[22] L.L. Frankfurt et al., Phys. Rev. Lett. 84, 3045 (2000).

[23] M. Sargsian (private communication).

[24] J.L. Matthews and R.O. Owens, Nucl. Instrum. Methods 111, 157 (1973). 